\title{
Enhancement of mechanical properties of poly(vinyl chloride) with polymethyl methacrylate-grafted halloysite nanotube
}

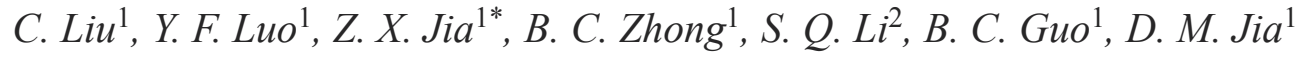 \\ ${ }^{1}$ College of Materials Sci. and Eng., South China University of Technology, Guangzhou 510640, PR China \\ ${ }^{2}$ XinJiang Tianye Group Co., Ltd., Xinjiang, Shihezi 832014, PR China
}

Received 13 November 2010; accepted in revised form 10 January 2011

\begin{abstract}
Halloysite nanotubes(HNTs) grafted with Polymethyl methacrylate(PMMA) were synthesized via radical polymerization. The properties of PMMA-grafted HNTs were characterized by transmission electron microscopy (TEM), fourier transform infrared spectroscopy (FTIR), thermogravimetric analysis (TGA) and X-ray photoelectron spectroscopy (XPS). The results showed that PMMA grafted to the surfaces of HNTs successfully. Then, PVC/PMMA-grafted HNTs nanocomposites were prepared by melt compounding. The morphology, mechanical properties and thermal properties of the nanocomposites were investigated. PMMA-grafted HNTs can effectively improve the toughness, strength and modulus of PVC. The glass transition and thermal decomposition temperatures of PVC phase in PVC/PMMA-grafted HNTs nanocomposites are shifted toward slightly higher temperatures. The grafted HNTs were uniformly dispersed in PVC matrix as revealed by TEM photos. The fracture surfaces of the nanocomposites exhibited plastic deformation feature indicating ductile fracture behaviors. The improvement of toughness of PVC by PMMA-grafted HNTs was attributed to the improved interfacial bonding by grafting and the toughening mechanism was explained according to the cavitation mechanism.
\end{abstract}

Keywords: nanocomposites, halloysite nanotubes, poly(vinyl chloride), surface modification, polymethyl methacrylate

\section{Introduction}

Poly(vinyl chloride) (PVC) is an important commercial thermoplastic, which is widely used in industrial fields due to its good properties and lowcost. However, its brittleness, low thermal stability and poor processability limit its application. Incorporation of nanopaticles into PVC to form nanocomposite is an effective method for improving the mechanical and thermal properties of PVC. For example, the addition of organic montmorillonite (MMT) within $0.5-3 \mathrm{wt} \%$ content can enhance the mechanical properties and improve the processing stability of the PVC/MMT nanocomposites [1]. Calcium carbonate $\left(\mathrm{CaCO}_{3}\right)$ nanoparticles stiffen and toughen $\mathrm{PVC}$ simultaneously in $\mathrm{PVC} / \mathrm{CaCO}_{3}$ nanocomposites which were synthesized by in situ polymerization [2]. Wang et al. [3] prepared PVC composites with poly caprolactone (PCL) modified multiwall carbon nanotube (MWNT) and found that MWNT-PCL can increase the Young's modulus, tensile strength and roughness of PVC.

Due to the strong interactions among the nanoparticles, uniformly dispersion of them in polymers is hard to achieve. Numerous methods have been employed to improve the dispersion of nanoparticles in polymer matrix. Surface modification of the nanoparticle via grafting is one of the most common methods for increasing the compatibility

\footnotetext{
${ }^{*}$ Corresponding author, e-mail: zxjia@scut.edu.cn
}

(c) BME-PT 
between nanoparicles and polymers. The interfacial bonding between the nanoparticles and the matrix can be enhanced by improved miscibility and entanglement of the grafting polymers with the matrix macromolecules. The modified nanoparticles can be uniformly dispersed in polymer matrix. Xie et al. [4] treated the nano-sized antimony trioxide $\left(\mathrm{Sb}_{2} \mathrm{O}_{3}\right)$ particles by in-situ methyl methacrylate (MMA)/ $\mathrm{Sb}_{2} \mathrm{O}_{3}$ polymerization and then prepared $\mathrm{PVC} / \mathrm{Sb}_{2} \mathrm{O}_{3}$ nanocomposites. They found that modified $\mathrm{Sb}_{2} \mathrm{O}_{3}$ could effectively reinforce and toughen PVC due to the improved interfacial bonding. Nanosilica and talc can also be grafted by PMMA for the purposed of toughening PVC [5, 6].

Halloysite nanotubes (HNTs) are a type of natural aluminoslicate nanotube and can be used as nanofiller for polymers, such as polypropylene [7, 8], epoxy resin[9-11], polyethylene [12], polyamide [13, 14] and rubber [15-17]. Due to their unique structure, HNTs show promising reinforcing effect for polymers. For example, the strength and modulus of polymers can be significantly improved by HNTs. HNTs can also enhance the thermal stability of polymers. Recently, Mondragon et al. [18] found that HNTs could increase the elongation of PVC indicating their toughening effect for PVC. In virtue of the poor compatibility between HNTs and PVC, surface treatment of HNTs before adding them to PVC is necessary.

Recently, the modification of HNTs has attracted great attention of scientists $[19,20]$. In this study, HNTs grafted with polymethyl methacrylate (PMMA), a polar polymer which has been proved with excellent compatibility with PVC, have been prepared. Then the grafted HNTs were compounded with PVC for preparing PVC/PMMA-grafted HNTs nanocomposites. The surface properties of HNTs before and after grafting were characterized. The morphology, mechanical properties and thermal properties of the nanocomposites have been investigated in detail. It is expected that the improved interfacial bonding by grafting may significantly enhance the mechanical properties of the nanocomposites.

\section{Experimental}

\subsection{Materials}

Halloysite nanotubes (HNTs), collected from Hubei Province, China, were purified according to the reported method [11]. PVC (SG-5) with an average polymerization degree between 981 and 1135 was a powder product offered by Wuhai Chemical Co., Ltd, China. Rare earth complex heat stabilizer (WWP-C) was supplied by Guangdong Winner Functional Materials Co. Ltd., China. Di(2-ethylhexyl) phthalate (DOP), chemically pure grade, was supplied by Tianjing Damao Chemical Reagent Factory, China. Methyl methacrylate (MMA), chemically pure grade, was produced by Tianjin Damao Chemical Reagent Factory, China. MMA was distilled under reduced pressure before being used to remove the inhibitor. 3-(Trimethoxysilyl)-propyl methacrylate (MPS), chemically pure grade, was produced by Nanjing Shuguang Chemical group Co., Ltd. Sodium dodecyl sulfate (SDS), analytical reagent, was produced by Tianjin Qilun tech Co., Ltd. Potassium persulfate (KPS) analytical reagent, was produced by Tianjin Fuchen Chemical Reagent Factory, China. Deionized water was applied for all polymerization and treatment processes.

\subsection{Preparation of PMMA-grafted HNTs}

HNTs were firstly modified by MPS coupling agent. The required volumes of MPS were added into the toluene dispersions containing $\mathrm{HNTs}$ at $110^{\circ} \mathrm{C}$ under stirring and agitate for $10 \mathrm{~h}$. After drying at $50^{\circ} \mathrm{C}$ under vacuum for $24 \mathrm{~h}$, the MPS-functionalized HNTs were extracted by a Soxhlet extractor for $12 \mathrm{~h}$ with toluene. The product was finally dried at $50^{\circ} \mathrm{C}$ under vacuum condition for $24 \mathrm{~h}$. The amount of MPS grafted on HNTs surfaces was evaluated by thermal gravimetric analysis (TGA).

The polymerization of MMA in the presence of MPS-functionalized HNTs was carried out in a four necked flask equipped with a reflux condenser, a stirrer, a thermometer and nitrogen inlet. First, the functionalized HNTs were dispersed in water containing SDS ultrasonically, and then heated under constant stirring with protection of nitrogen flow. When the temperature reached around $80^{\circ} \mathrm{C}$, the aqueous solution of initiator KPS was added. After $10 \mathrm{~min}$, a continuous dosage of $10 \mathrm{wt} \% \mathrm{MMA}$ (based on HNTs) was added and reacted at $80^{\circ} \mathrm{C}$ for 3 hour. The polymerization products were extracted with chloroform for $24 \mathrm{~h}$ in a Soxhlet apparatus to remove the ungrafted polymer after emulsion breakage and purification process. The obtained PMMA-grafted HNTs were dried at $50^{\circ} \mathrm{C}$ to a constant weight under vacuum. 


\subsection{Preparation of PVC/PMMA-grafted HNTs nanocomposites}

The PVC nanocomposites were formulated with $5 \mathrm{phr}$ heat stabilizer and $5 \mathrm{phr}$ of DOP, as well as various contents of PMMA-grafted HNTs. The ingredients were first mixed in a high-speed rotating mixer and then plasticized by a two-roll mill at $175^{\circ} \mathrm{C}$ for $8 \mathrm{~min}$. The compounds were then compression molded to plates at $175^{\circ} \mathrm{C}$ for 5 min with a 5 min preheating period. Specimens of suitable dimensions for mechanical testing were cut from the compression molded plates.

\subsection{Characterization}

Fourier Transform Infrared Spectroscopy (FTIR)

A Bruker Vector 33 FTIR spectrometer (Germany) was used for FTIR analysis. The infrared spectra were recorded in absorbance units in the 4000$400 \mathrm{~cm}^{-1}$ range. FTIR spectra were measured in $\mathrm{KBr}$ pellets containing $1 \%$ finely ground samples.

$X$-ray Photoelectron Spectroscopy (XPS)

XPS analyses were performed using a Kratos Axis Ultra (DLD) (England) with an aluminum (mono) $\mathrm{K}_{\alpha}$ source $(1486.6 \mathrm{eV})$.

Thermogravimetric Analysis (TGA)

TGA was conducted under nitrogen atmosphere with Netzsch TG $209 \mathrm{~F}$ (Germany) at a heating rate of $10^{\circ} \mathrm{C} / \mathrm{min}$ from $30^{\circ} \mathrm{C}$ to $700^{\circ} \mathrm{C}$.

The grafting percentage onto the surface of HNTs was calculated from the TGA analysis by the Equation (1):

Percentage of grafting $[\%]=$

$$
=\frac{\text { Polymer grafted }[\mathrm{g}]}{\text { HNTs used }[\mathrm{g}]} \cdot 100
$$

\footnotetext{
Mechanical properties

Zwick/Roell Z010 and Zwick pendulum 5113 testing machines (Germany) were used to perform the tensile, flexural and impact testing according to ISO 527:1993, ISO 178:1993 and ISO 180:1993, respectively.

Scanning Electron Microscopy (SEM)

The fracture surfaces of impact samples were coated with a thin layer of gold before any observations. The fracture surface was then examined by Nova Nano SEM 430 machine (FEI, Netherlands). The voltage of the electron beam used for SEM observation was $10 \mathrm{kV}$.
}

\section{Transmission Electron Microscope (TEM)}

The morphologies of HNTs and nanocomposites were characterized with a Philip Tecnai 12 TEM (Eindhoven, Netherlands). The TEM samples of the nanocomposites were cut into thin pieces of about $120 \mathrm{~nm}$ in thickness with an ultramicrotome (EMUC6, Leica, Germany) before observation.

Dynamic Mechanical Analysis (DMA)

DMA was conducted with a EPLEXOR dynamic mechanical analyzer (Gabo Qualimeter Testanlagen $\mathrm{GmbH}$; Ahlden, Germany) at an oscillation frequency of $1.0 \mathrm{~Hz}$. The temperature range was from 30 to $150^{\circ} \mathrm{C}$ with a heating rate of $3^{\circ} \mathrm{C} / \mathrm{min}$.

Differential Scanning Calorimetry (DSC)

The glass transition temperature $\left(T_{\mathrm{g}}\right)$ was evaluated by using DSC method with a NETZSCH Instruments DSC 204 F1 (Germany), operated in nitrogen at a rate of $10 \mathrm{C} / \mathrm{min}$.

\section{Results and discussion}

\subsection{Properties of PMMA-grafted HNTs}

As shown in TEM images (Figures 1a and b), the HNTs used in this study exhibit mainly tubular shapes with an outer diameter of $20 \sim 50 \mathrm{~nm}$. The hollow lumen of the HNTs is clearly visible in Figure 1b. TEM images of PMMA-grafted HNTs are shown in Figures $1 \mathrm{c}$ and d. It can be seen that the surface of the PMMA-grafted HNTs is rougher than that of pristine HNTs. Some irregular precipitate is found on the outer surface of the PMMA-grafted HNTs. A part of hollow lumen of HNTs is filled after grafting. This indicates that a layer of PMMA is wrapped on the outer-surface and inner-surface of HNTs.

Figure 2 shows FTIR spectra of the pristine HNTs, MPS-functionalized HNTs and PMMA-grafted HNTs. Compared to the spectrum of HNTs, the most significant feature of MPS-HNTs is the presence of characteristic band at 2950, 1720 and $1640 \mathrm{~cm}^{-1}$ which is ascribed to the $\mathrm{C}-\mathrm{H}$ stretching vibration, $\mathrm{C}=\mathrm{O}$ symmetric stretching vibration, and $-\mathrm{C}=\mathrm{C}$ vibration of MPS respectively. This indicates that the MPS grafts on the surface of HNTs successfully. For the spectrum of PMMA-grafted HNTs, the peak around $1732 \mathrm{~cm}^{-1}$ is ascribed to the $\mathrm{C}=\mathrm{O}$ vibration of PMMA. As the samples have been extracted, the physically absorbed MPS and PMMA have been removed. Therefore, PMMA are covalently bonded on the surfaces of HNTs through graft 


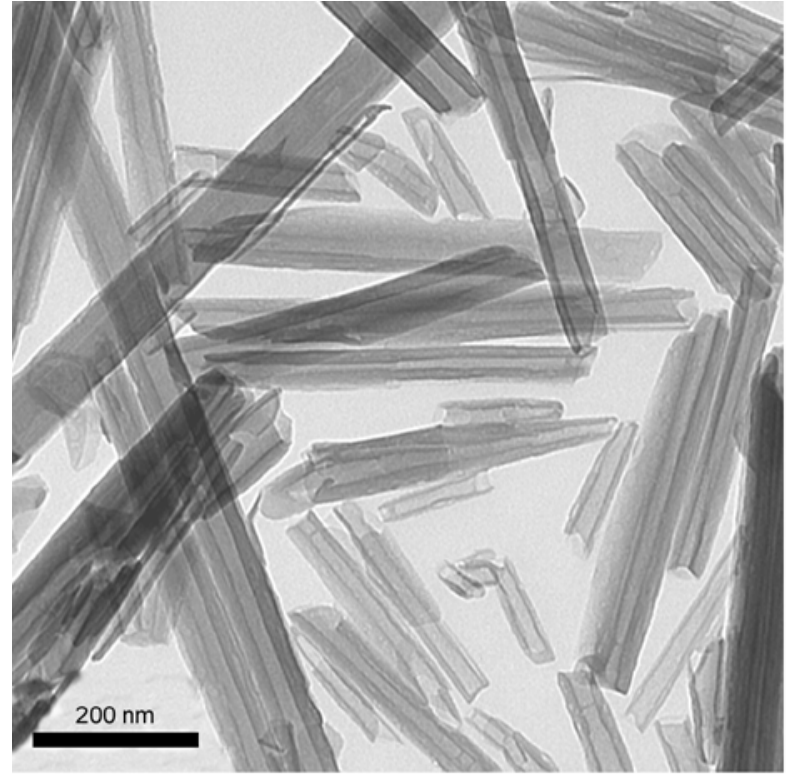

a)

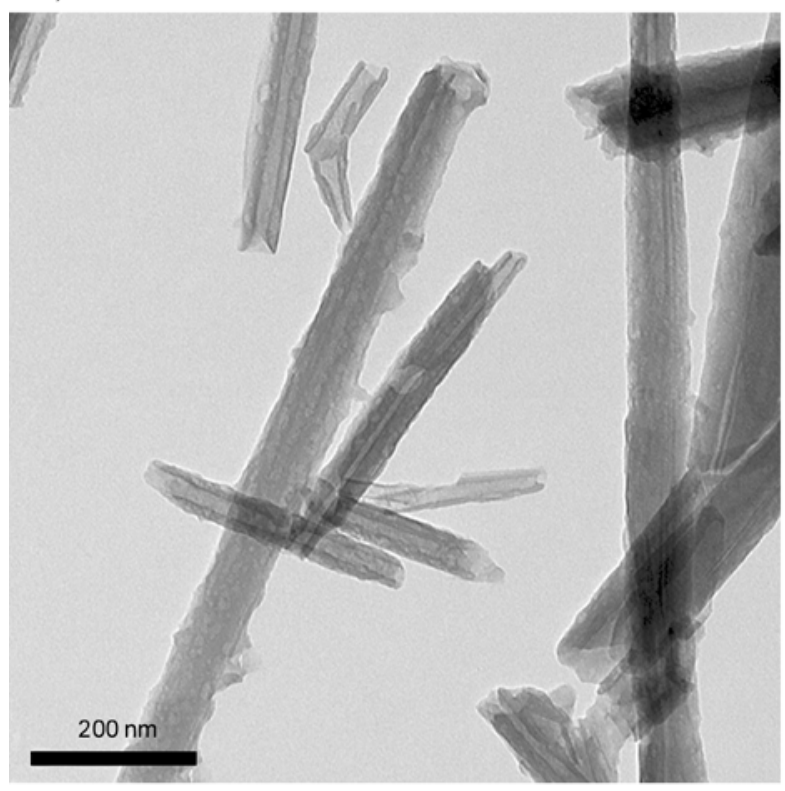

c)

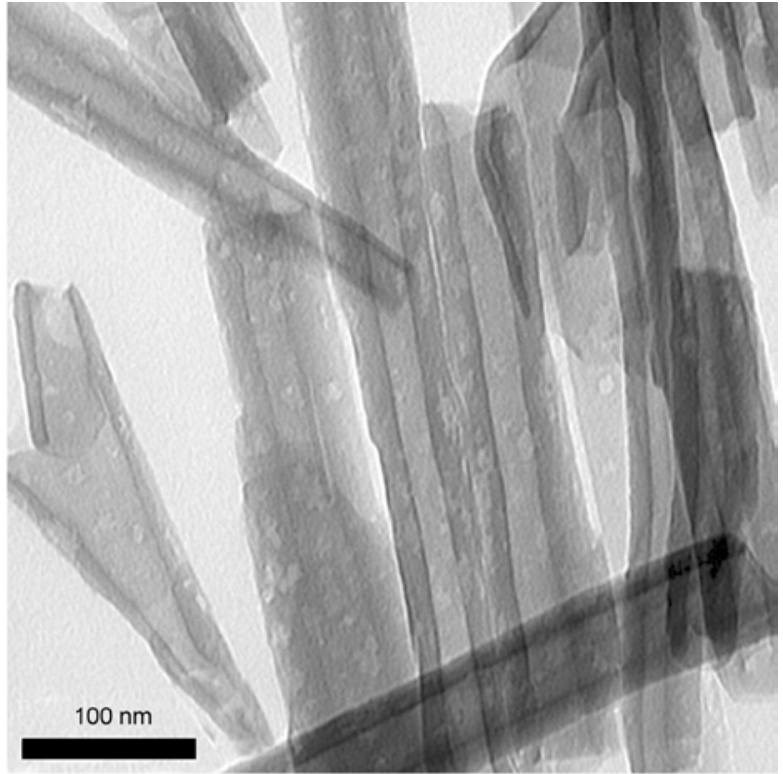

b)

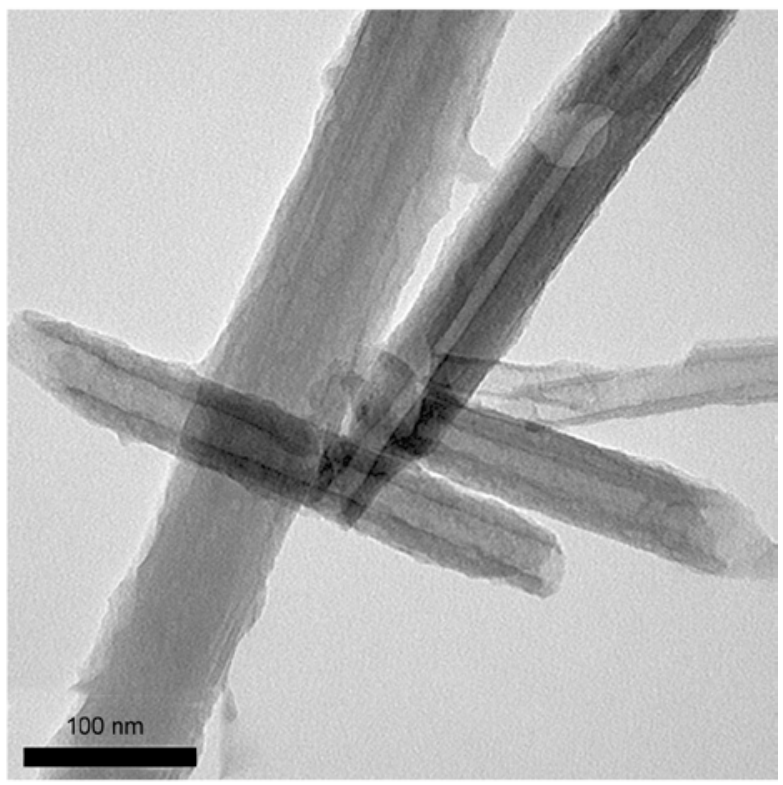

d)

Figure 1. TEM photographs of HNTs and PMMA-grafted HNTs. (a) and (b) HNTs; (c) and (d) PMMA-grafted HNTs

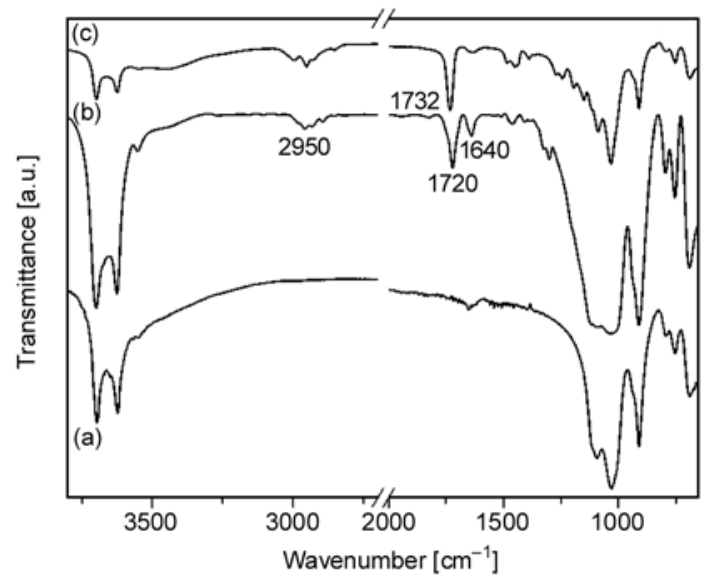

Figure 2. FT-IR spectra of HNTs and modified HNTs. (a) HNTs; (b) MPS-modified HNTs (c) PMMAgrafted HNTs polymerization. The content of PMMA bonded on the HNTs particles is $5.45 \mathrm{wt} \%$, as calculated from TGA.

Surface element analysis of XPS survey can give the detailed variations of element information on the particles surface qualitatively. The low-resolution XPS survey spectra of the HNTs and PMMAgrafted HNTs are shown in Figure 3. As illustrated in Figure 3a, XPS survey spectrum of HNTs shows the oxygen peak at $531 \mathrm{eV}$, carbon peak at $284 \mathrm{eV}$, silicon peak at $102 \mathrm{eV}$ and aluminum peak at $73 \mathrm{eV}$ [21]. Table 1 summarizes the characteristic XPS data. It can be seen that the detected relative con- 


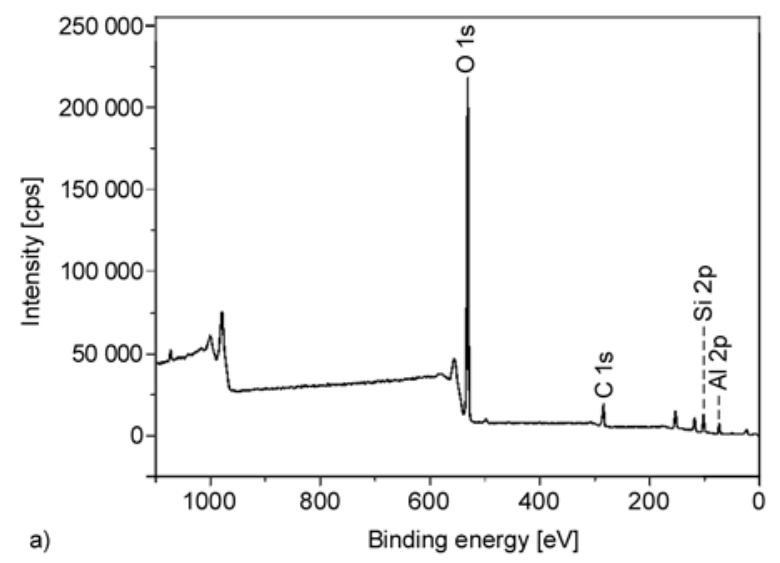

Figure 3. XPS spectra of HNTs (a) and PMMA-grafted HNTs (b)

Table 1. XPS atomic content [\%] for the HNTs and PMMAgrafted HNTs

\begin{tabular}{|c|c|c|}
\hline Atom & HNTs & PMMA-grafted HNTs \\
\hline $\mathrm{C}$ & 15.30 & 63.82 \\
\hline $\mathrm{O}$ & 54.33 & 22.07 \\
\hline $\mathrm{Al}$ & 13.72 & 5.78 \\
\hline $\mathrm{Si}$ & 16.66 & 8.34 \\
\hline
\end{tabular}

centration of carbon of PMMA-grafted HNTs is remarkably increased compared with that of HNTs. The relative concentrations of oxygen, aluminum and silicon are decreased. The result can be explained by the fact that grafting of PMMA introduces abundant carbon atoms onto the surfaces of HNTs. This result is consistent with the previous reported of silane modified HNTs [22]. The above XPS results also verify the successful modification of HNTs.

\subsection{Morphology of PVC/PMMA-grafted HNTs nanocomposites}

Dispersion of the fillers is one of the most critical issues in determining the mechanical properties of nanocomposites [23]. The agglomerated fillers in the polymer matrix may act as the stress-concentration points, leading to deteriorated properties [24]. The dispersion of the PMMA-grafted HNTs in the matrix was evaluated using TEM (Figure 4). For the nanocomposites with 1 phr PMMA-grafted HNTs (Figures $4 \mathrm{a}$ and b), a good dispersion is achieved. HNTs dissociate into isolated nanotubes, and no visible particle agglomerates are observed, indicating good compatibility between the grafted HNTs and PVC. Only a small amount of agglomerates are found in the nanocomposite with 5 phr PMMAgrafted HNTs (Figures 4c and d).

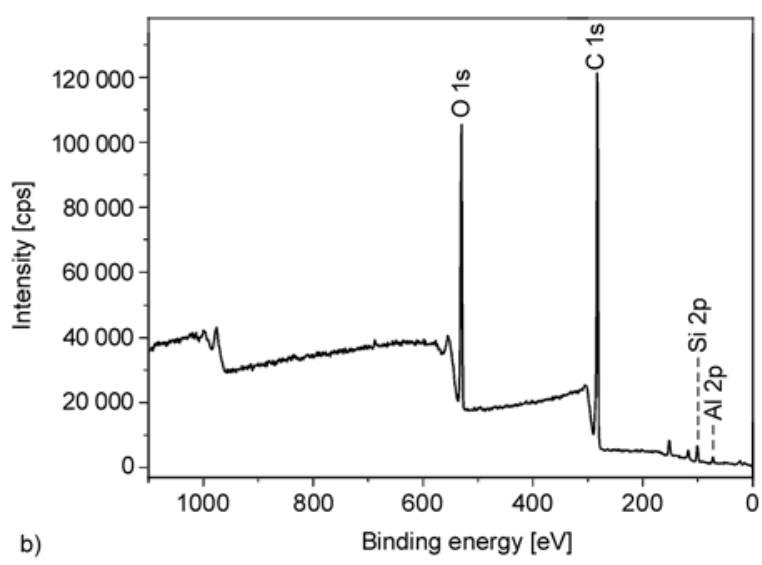

The morphology of the fracture surfaces for PVC and PVC/PMMA-grafted HNTs nanocomposites were determined by SEM. It can be seen that the fractured surface of the PVC (Figure 5a) is relatively smooth, showing brittle failure characteristics. In contrast, the fractured surfaces of the nanocomposite (Figures 5b, c and d) are uneven and consist of a large number of squamous structures, exhibiting plastic deformation characteristics. The overall size of the plastic deformation zone is related to the fracture toughness $[25,26]$. The SEM results indicate that the nanocomposites have higher toughness than pristine PVC.

\subsection{Dynamic mechanical properties of PVC/PMMA-grafted HNTs nanocomposites}

Dynamic mechanical analysis can be used to evaluate material stiffness at various temperatures. It is particularly useful in the characterization of materials used in applications above room temperature [27]. The variation of the storage modulus $\left(E^{\prime}\right)$ with temperature for PVC and PVC/PMMA-grafted HNTs nanocomposites are shown in Figure 6. The storage moduli of all of the PVC/PMMA-grafted HNTs nanocomposites are higher than those of PVC below the glass transition temperature $\left(T_{\mathrm{g}}\right)$. However, when the nanoparticle content is $5 \mathrm{phr}$, the storage modulus is progressively reduced with increasing temperature. It is known that the relative values of storage modulus of particulate-filled polymer composites are related to the stiffness and influenced by the effective interfacial interaction between the inorganic particles and polymer chains $[5,28,29]$. In general, the stronger effective interfa- 


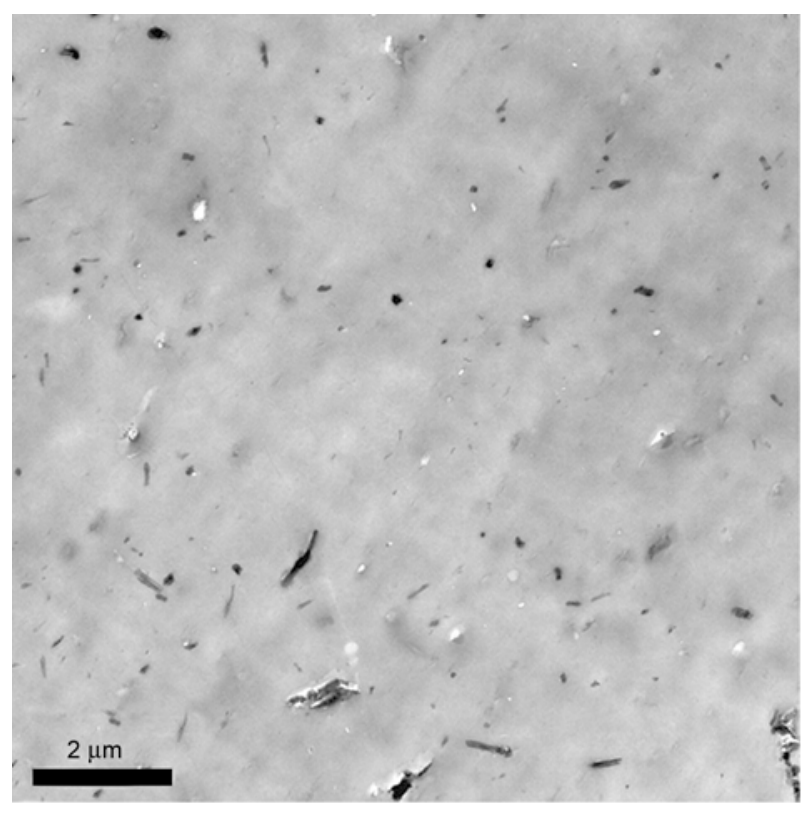

a)

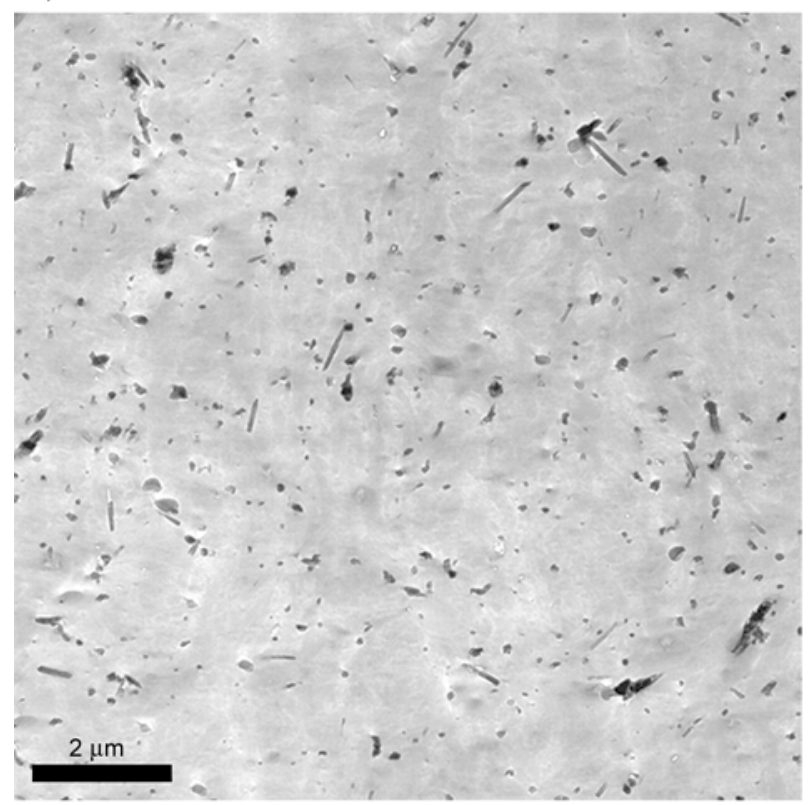

c)

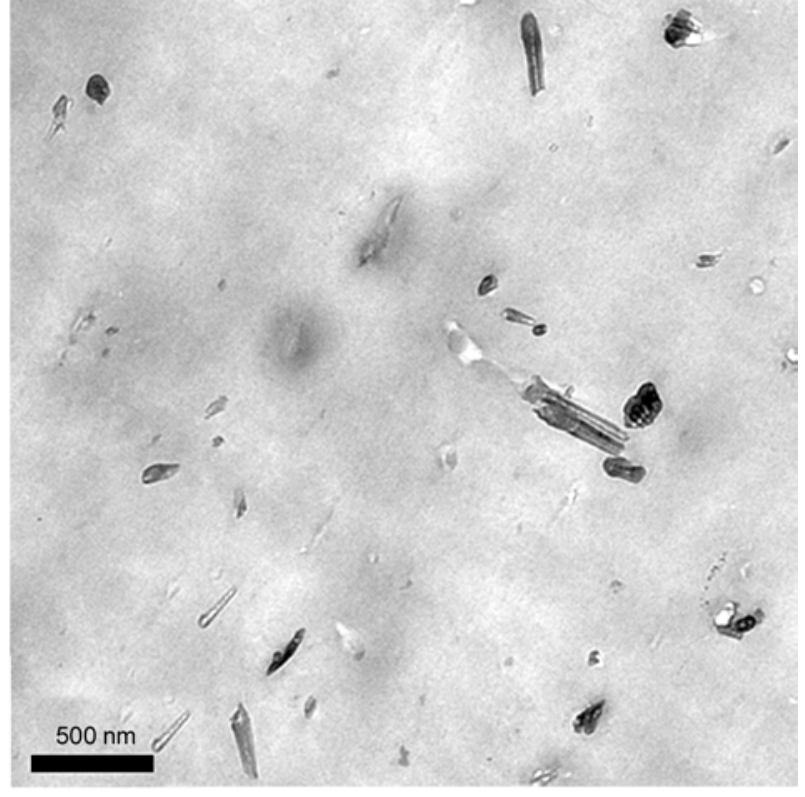

b)

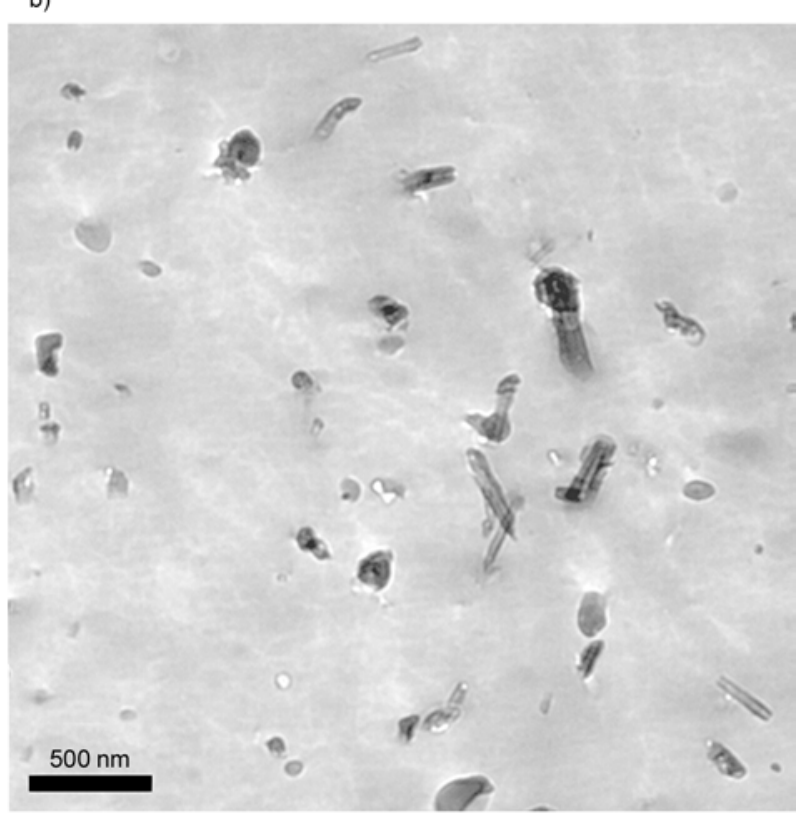

d)

Figure 4. TEM photographs of PVC/PMMA-grafted HNTs nanocomposites. (a) and (b) PVC/PMMA-grafted HNTs (100/1); (c) and (d) PVC/PMMA-grafted HNTs (100/5)

cial interaction between the matrix and the fillers is, the higher value of storage modulus of composites is [30]. The result indicates that PMMA-grafted HNTs can increase the stiffness of PVC. This is attributed to the following facts that there is strong effective interfacial interaction between the PVC matrix and PMMA-grafted HNTs while the welldispersed PMMA-grafted HNTs reduce mobility of PVC chains. When the nanoparticle content is $5 \mathrm{phr}$, agglomeration of PMMA-grafted HNTs weakens the interaction between PVC chains and nanoparticles so that the storage modulus decreases a little.

\subsection{Thermal properties of PVC/PMMA-grafted HNTs nanocomposites}

The influence of PMMA-grafted HNTs on the mobility of the PVC chains was further investigated by the DSC method for comparing the glass transition temperature $\left(T_{\mathrm{g}}\right)$. The glass transition temperatures $\left(T_{\mathrm{g}}\right)$ of the PVC phases in pristine PVC and PVC/PMMA-grafted HNTs nanocomposites are listed in Table 2. The $T_{\mathrm{g}}$ of PVC in nanocomposites tends to shift slightly towards to higher temperature than that of pristine PVC, indicating the change in 


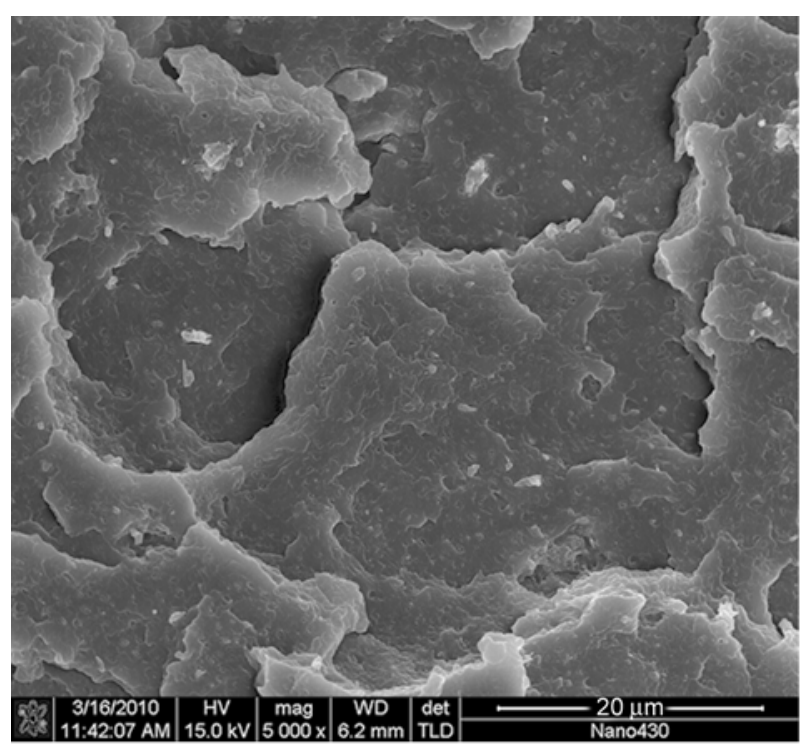

a)

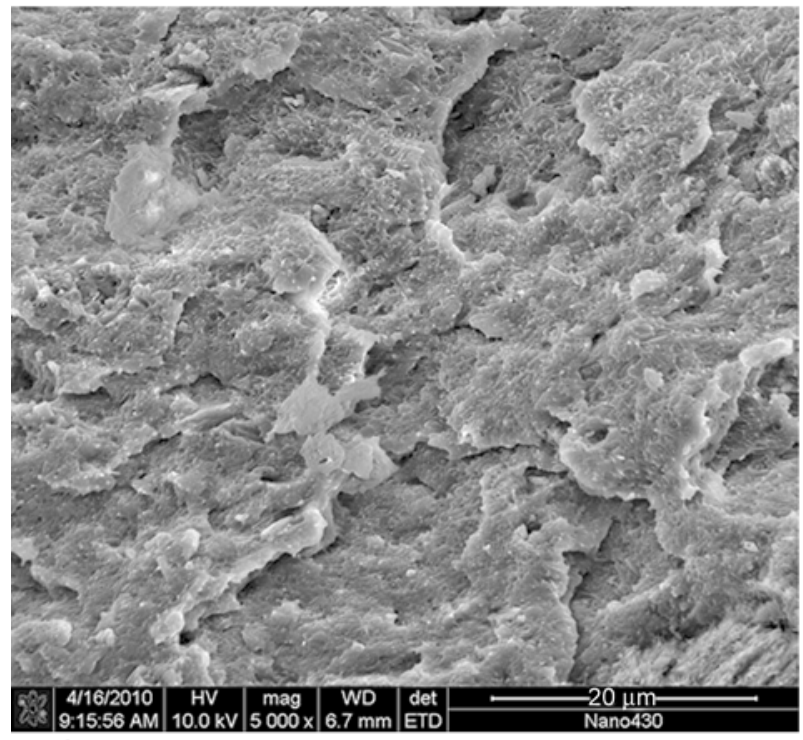

c)

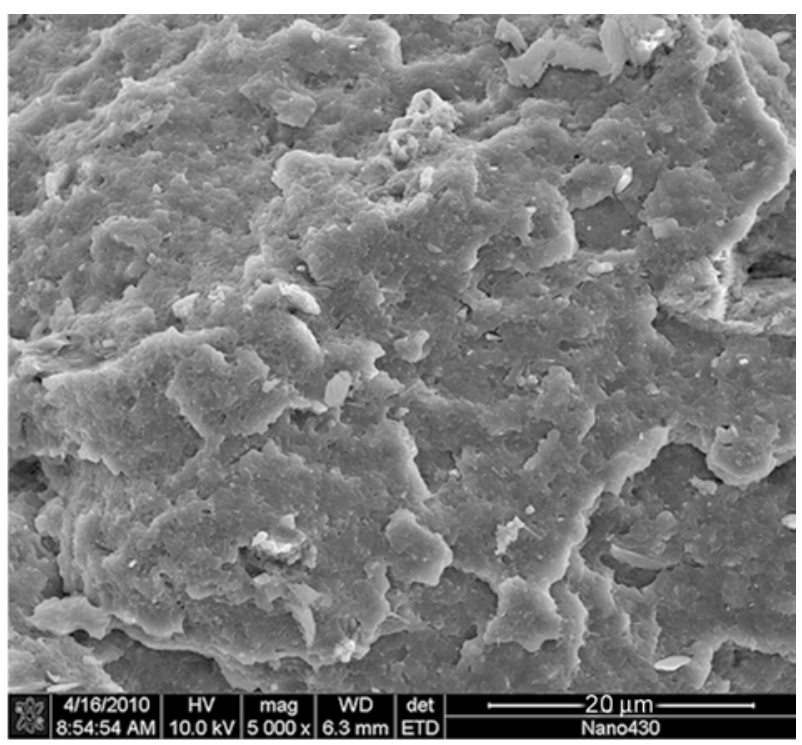

b)

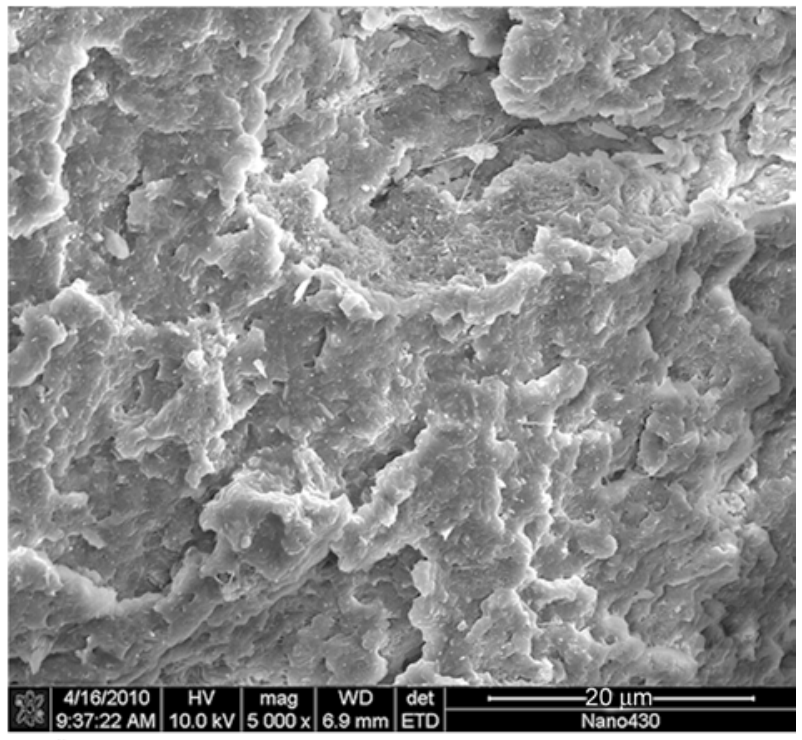

d)

Figure 5. SEM micrographs of the impact fracture surfaces of PVC and PVC/PMMA-grafted HNTs nanpcomposites. (a) PVC; (b) PVC/PMMA-grafted HNTs (100/1); (c) PVC/PMMA-grafted HNTs (100/3); (d) PVC/PMMAgrafted HNTs (100/5)

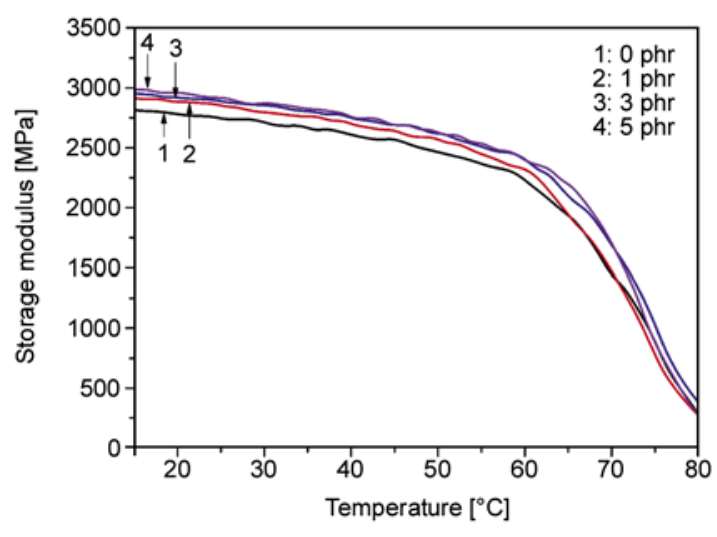

Figure 6. Storage modulus $\left(E^{\prime}\right)$ versus temperature for PVC and PVC/ PMMA-grafted HNTs nanpcomposites
Table 2. DSC and TGA results of PVC and PVC/PMMAgrafted HNTs nanocomposites

\begin{tabular}{|l|c|c|c|c|}
\hline \multicolumn{1}{|c|}{ Sample } & $\begin{array}{c}\mathbf{T}_{\mathbf{g}} \\
{\left[{ }^{\circ} \mathbf{C}\right]}\end{array}$ & $\begin{array}{c}\mathbf{T}_{\mathbf{5}} \mathbf{6} \\
{\left[{ }^{\circ} \mathbf{C}\right]}\end{array}$ & $\begin{array}{c}\mathbf{T}_{\mathbf{5 0}} \\
{\left[{ }^{\circ} \mathbf{C}\right]}\end{array}$ & $\begin{array}{c}\mathbf{T}_{\mathbf{m a x}} \\
{\left[{ }^{\circ} \mathbf{C}\right]}\end{array}$ \\
\hline Pristine PVC & 66 & 234 & 290 & 263 \\
\hline PVC/PMMA-grafted HNTs (100/1) & 71 & 236 & 291 & 265 \\
\hline PVC/PMMA-grafted HNTs (100/3) & 72 & 239 & 295 & 267 \\
\hline PVC/PMMA-grafted HNTs (100/5) & 73 & 237 & 295 & 266 \\
\hline
\end{tabular}

the polymer thermodynamics. The $T_{\mathrm{g}}$ of $\mathrm{PVC}$ increases to 73 from $66^{\circ} \mathrm{C}$ by adding $5 \mathrm{phr}$ of PMMA-grafted HNTs. This behavior is similar to what is observed in $\mathrm{PVC} / \mathrm{Sb}_{2} \mathrm{O}_{3}$ nanocomposites [4]. The PMMA shell on particles increases the 


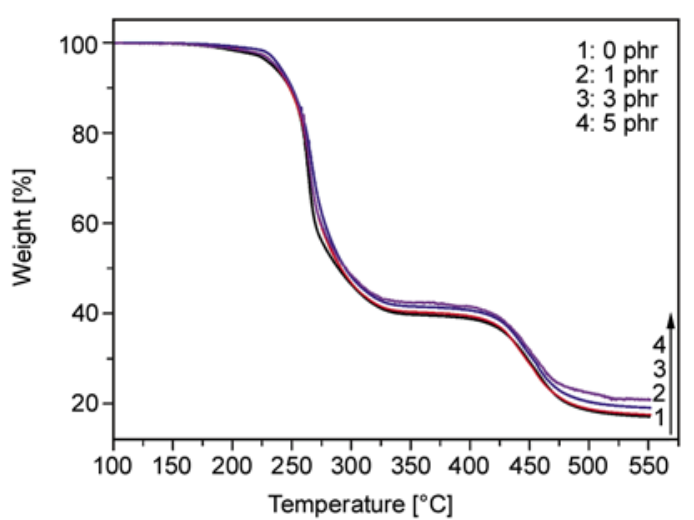

Figure 7. TGA curves of PVC and PVC/PMMA-grafted HNTs nanocomposites in nitrogen

interaction between the particles and PVC matrix and the particles restrain the thermal motion of PVC molecular chains, these lead to the enhancement of thermal stability of PVC.

The thermogravimetric (TG) curves of PVC and PVC/PMMA-grafted HNTs nanocomposites are presented in Figure 7. The first degradation stage which is observed in the temperature range of 250 $400^{\circ} \mathrm{C}$ is assigned to the progressive dehydrochlorination of PVC and the formation of conjugated poly-
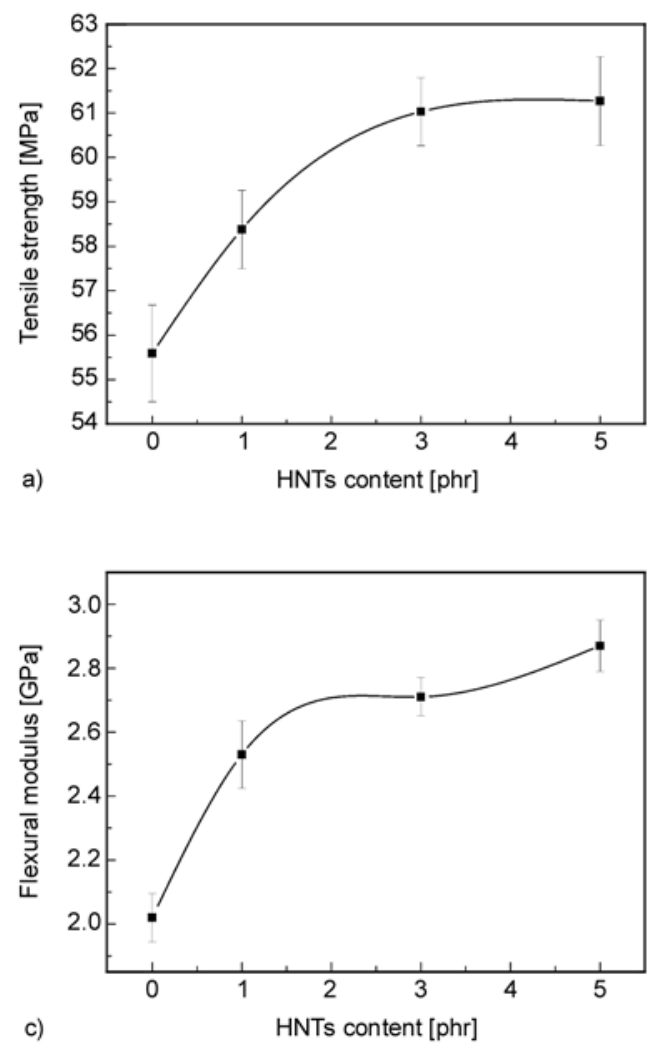

ene structure [31]. The temperatures of onset decomposition (the $5 \%$ weight loss temperature, $T_{5 \%}$ ), $50 \%$ weight loss $\left(T_{50 \%}\right)$, and fastest degradation ( $T_{\max }$, which is defined as the peak on the derivation of the TG curve) are summarized in Table 2. It can be seen that the $T_{5 \%}, T_{50 \%}$ and $T_{\max }$ of PVC/PMMAgrafted HNTs nanocomposites are slightly higher than that of pristine PVC. The well dispersed HNTs restrict the long-range-chain mobility of $\mathrm{PVC}$ and they can act as a barrier to hinder the permeability of volatile degradation products out of the material. These lead to the increase of the thermal stability of PVC. It seems that different nanoparticles have varied effect on the thermal stability of PVC. For example, the addition of $\mathrm{CaCO}_{3}$ can also lead to the slightly increased thermal stability of PVC, which is consistent with the present result [2]. But for the PVC/MMT nanocomposites prepared by in-situ polymerization, the onset decomposition temperatures of the PVC decreased by MMT, while the most rapid decomposition temperatures are enhanced [32]. From TGA result, it can be concluded that PVC/PMMA-grafted nanocomposites have good thermal stability.
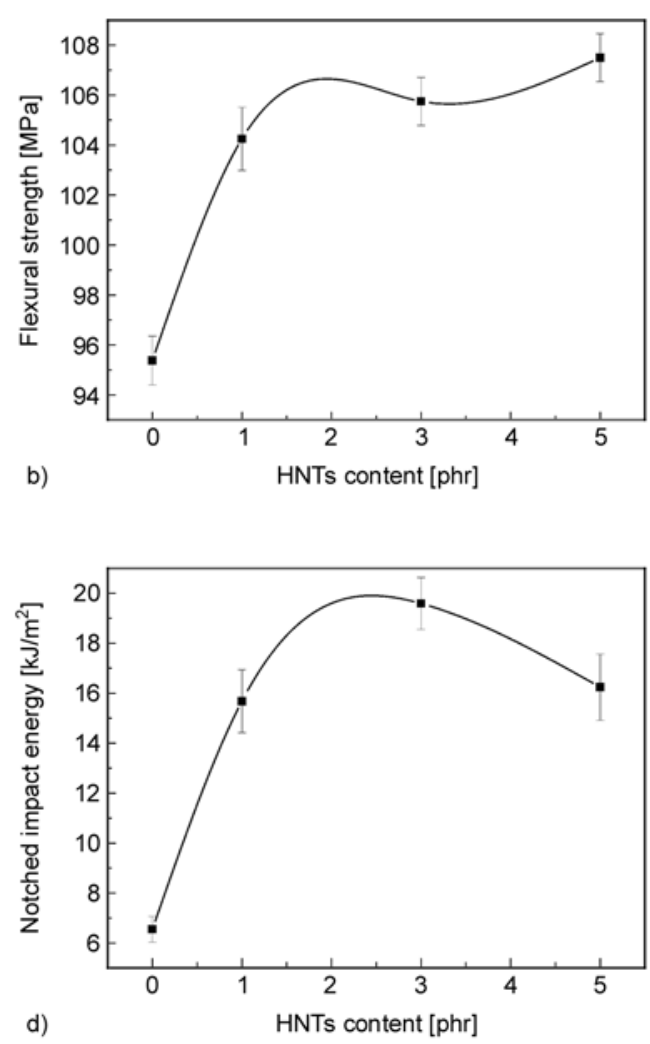

Figure 8. Effects of PMMA-grafted HNTs content on the mechanical properties of PVC/PMMA-grafted HNTs nanocomposites. (a) Tensile strength; (b) flexural strength; (c) flexural modulus; (d) Izod impact strength 


\subsection{Mechanical properties of PVC/PMMA-grafted HNTs nanocomposites}

The mechanical properties of PVC/PMMA-grafted HNTs nanocomposites at different contents of PMMA-grafted HNTs are shown in Figure 8. The tensile strength, flexural strength and flexural modulus of PVC/PMMA-grafted HNTs nanocomposites are remarkably increased with increasing PMMAgrafted HNTs content (Figures 8a, b and c). Comparing with unfilled PVC, the tensile strength, flexural strength and flexural modulus of nanocomposites filled with $5 \mathrm{phr}$ PMMA-grafted HNTs increases by $10,12.7$ and $42 \%$ respectively. The notched Izod impact strength of PVC/PMMA-grafted HNTs nano-

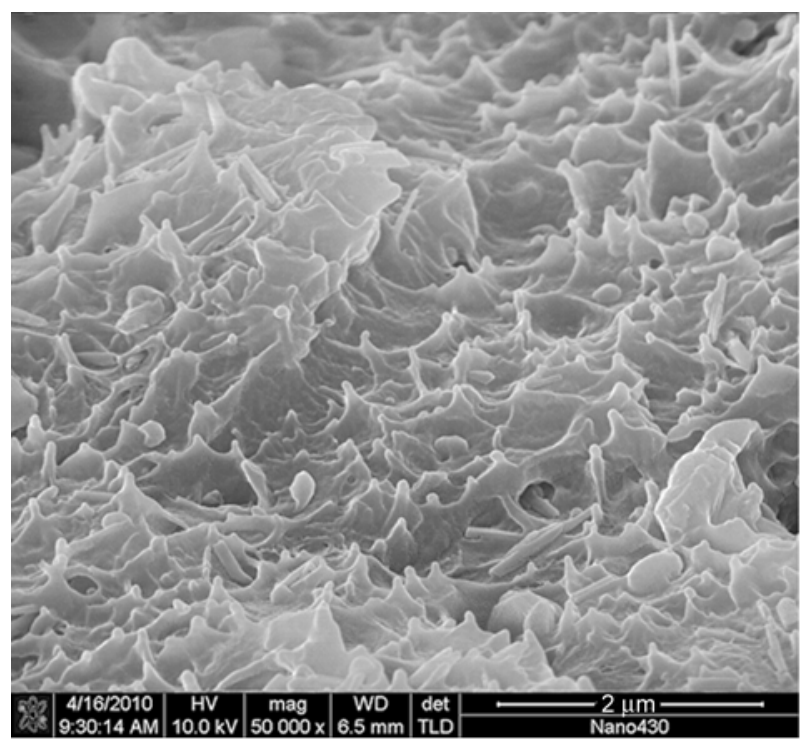

a)

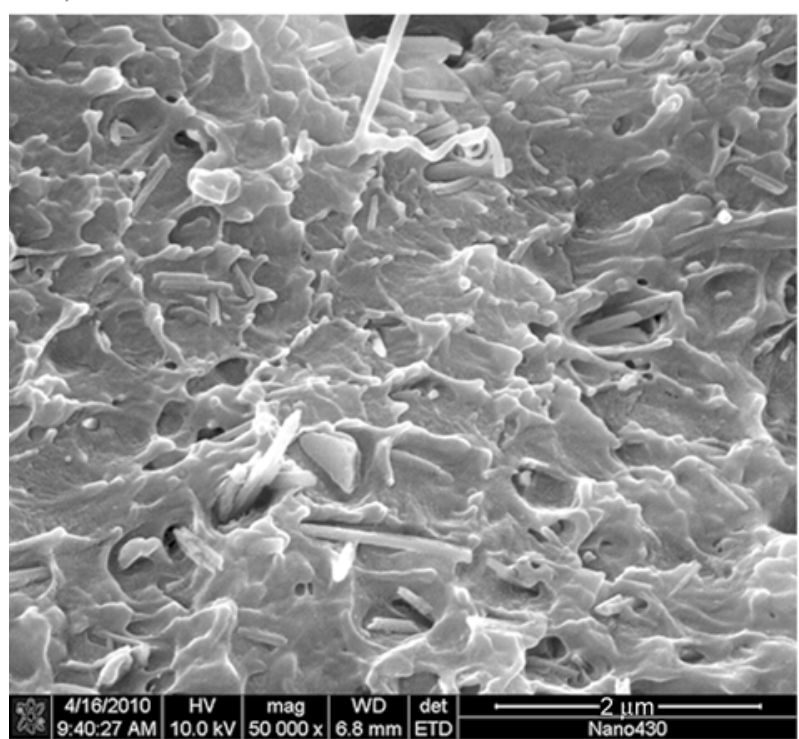

c) composites firstly increased with the loading of HNTs (Figures 8d). When the content of PMMAgrafted HNTs is $3 \mathrm{phr}$, the notched Izod impact strength of the nanocomposites is maximum, nearly double of that of pristine PVC. As the PMMAgrafted HNTs are increased to $5 \mathrm{phr}$, the notched Izod impact strength of the nanocomposites is a little decreased. This can be attributed to the agglomeration of HNTs. The mechanical properties results indicate that PVC can be reinforced and toughened by PMMA-grafted HNTs simultaneously.

\subsection{Toughening mechanism}

To investigate the toughening mechanism, the fracture surfaces of the nanpcomposites filled with $3 \mathrm{phr}$

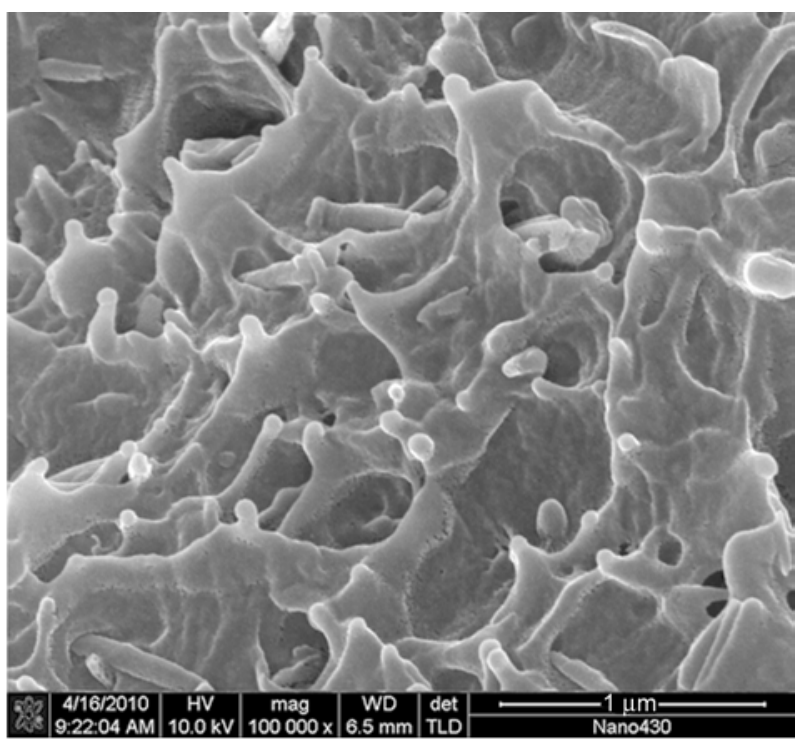

b)

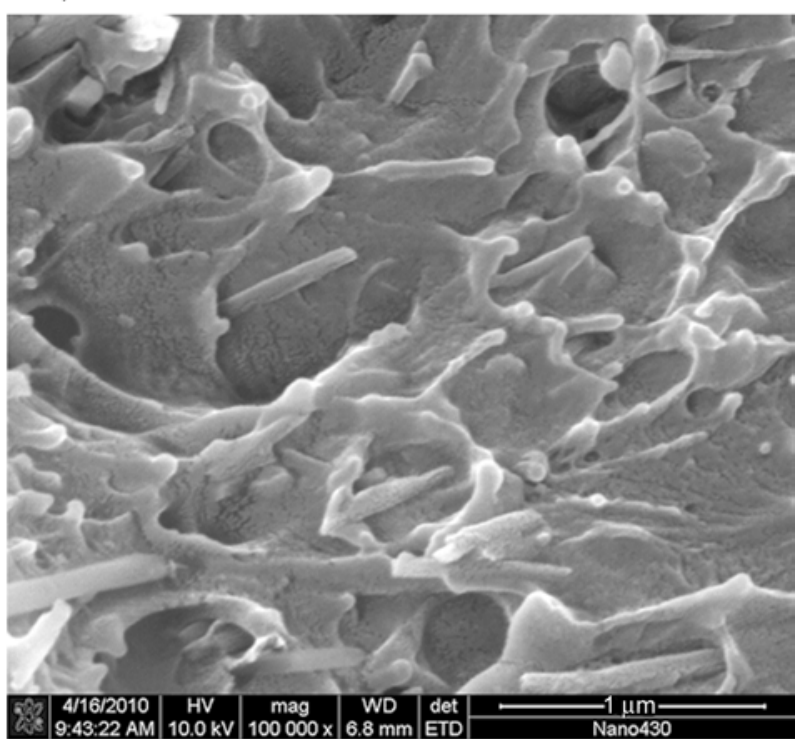

d)

Figure 9. SEM micrographs of the impact fracture surfaces of PVC/PMMA-grafted HNTs nanpcomposites. (a) and (b) PVC/PMMA-grafted HNTs (100/3); (c) and (d) PVC/PMMA-grafted HNTs (100/5) 
(Figures 9a and b) and $5 \mathrm{phr}$ (Figures 9c and d) PMMA-grafted HNTs were examined using a high resolution SEM. As revealed in Figure 9, the debonding and pull-out of HNTs nanoparticles are clearly shown in the fracture surface, and the PVC matrix is severely drawn around the nanoparticles. According to the cavitation mechanism [33], the particles debond prior to the yield strain of the matrix polymer, lead to the debonding at the nanoparticle-polymer interface, then the matrix is highly deformed and drawn around the particles, which dissipates impact energy and toughens the nanocomposites. The PMMA-grafted HNTs have good compatibility with PVC matrix and disperse homogeneous in the PVC matrix. Therefore, stress can be transferred to

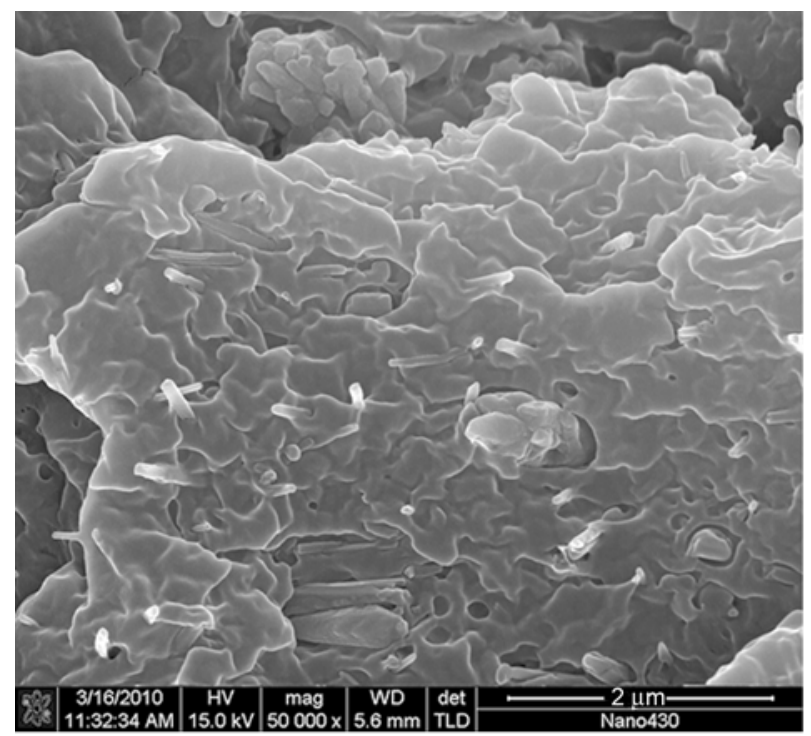

a) the stronger inorganic phase when the PVC matrix is subjected to applied force, so reinforcing and toughening effects can be observed at very low PMMA-grafted HNTs content. Comparing Figure $9 \mathrm{~b}$ with Figure 9d, it can be seen that the PMMAgrafted HNTs arrange more regularly and disperse more homogeneously in Figure 9b. While in Figure 9d, some HNTs arrange disorderly and some agglomeration can be observed. The agglomeration of HNTs weakens the interaction between PVC chains and HNTs. This is the reason why the toughness of the nanocomposite containing 5 phr PMMAgrafted HNTs slightly decreases comparing with that of the nanocomposites with 3 phr PMMAgrafted HNTs.

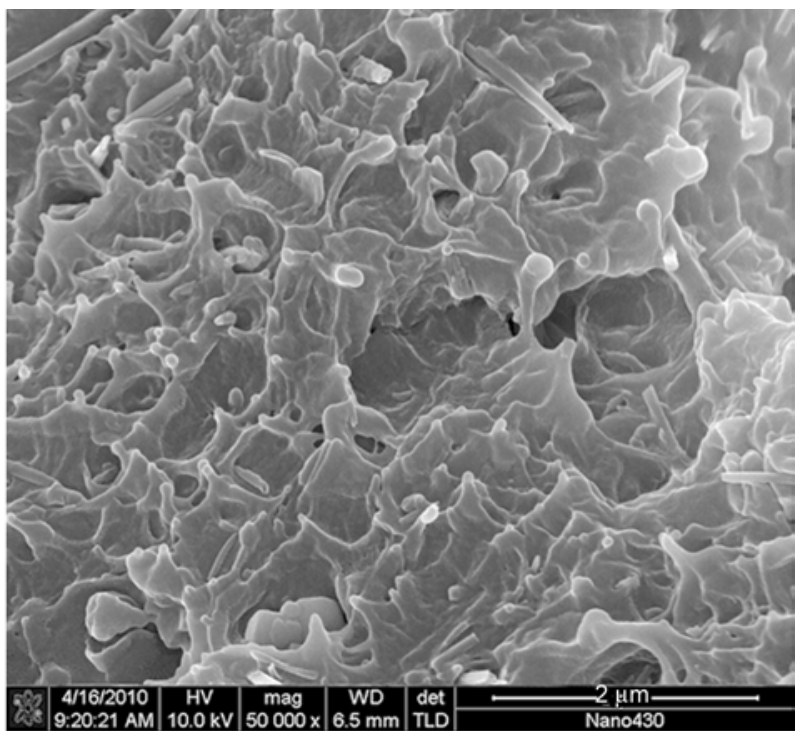

b)

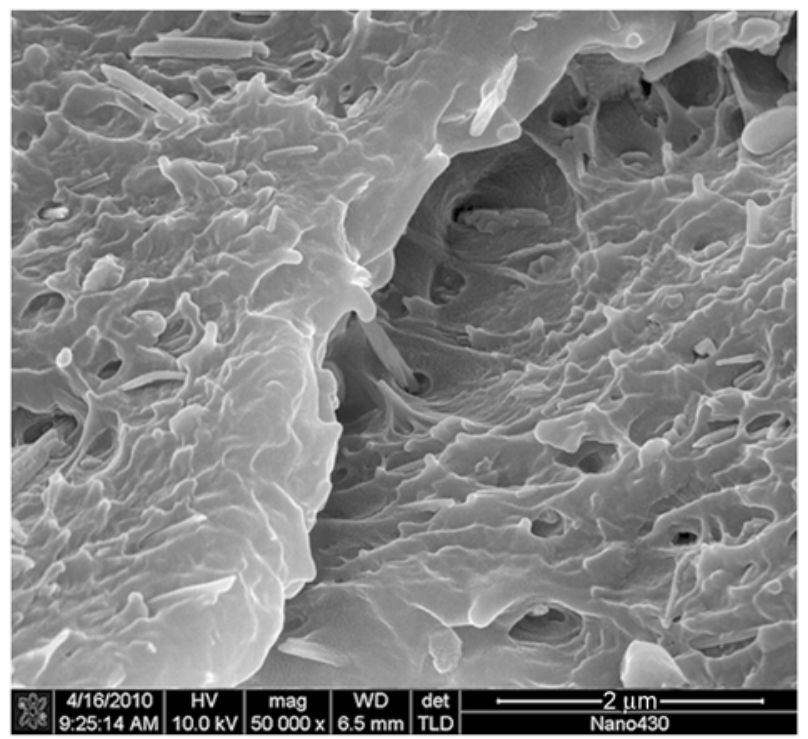

c)

Figure 10. SEM micrographs of the impact fracture surfaces of PVC/HNTs and PVC/PMMA-grafted HNTs nanpcomposites. (a) PVC/HNTs (100/3); (b) and (c) PVC/PMMA-grafted HNTs (100/3) 
To investigate the effect of PMMA shell covered on the surface of particles, the fracture surfaces of PVC/HNTs nanpcomposites and PVC/PMMAgrafted HNTs nanpcomposites with 3 phr particles loading were compared using a high resolution SEM (Figure 10a for the PVC/HNTs nanpcomposites and Figures $10 \mathrm{~b}$ and $\mathrm{c}$ for the PVC/PMMAgrafted HNTs nanpcomposites). For Figure 10b, the rough fracture surface, the uniform dispersion of PMMA-grafted HNTs and high plastic deformation around the PMMA-grafted HNTs are observed. While for Figure 10a, the fracture surface is not so rough and the deformation is not as severe as that of Figure 10b. This is because PMMA is miscible with PVC thermodynamically [34], the solubility parameter of PMMA is $9.4\left(\mathrm{~J} / \mathrm{cm}^{3}\right)^{1 / 2}$ similar to that of PVC which is $9.5\left(\mathrm{~J} / \mathrm{cm}^{3}\right)^{1 / 2}$. So PMMA shell covered on the surface of HNTs particle increases the interaction of the HNTs with PVC matrix. Besides, the surface energy of HNTs is decreased after surface grafting. The dispersion of HNTs is improved, and the agglomeration is reduced. The good interfacial bonding makes the load transfer from the matrix to the rigid inorganic phase effectively. The nanoparticles-polymer interfaces are debonded and consume impact energy. In Figure 10c the crack can be observed, besides, the surfaces of the crack are uneven and the HNTs bridge the gap. This is similar to the observation about epoxy/HNT nanocomposite [10]. Because of the strong interfacial bonding between PMMA-grafted HNTs and PVC, the cracks can be stabilized by the nanotube bridging and stopped developing into large and harmful cracks. When the crackopening force exceeded their fracture strength the HNTs would be broken, which consumed the impact energy. Therefore, the PVC/ PMMA-grafted nanocomposites exhibit high toughness.

\section{Conclusions}

PMMA-grafted HNTs synthesized by grafting methyl methacrylate on the surfaces of HNTs. The FTIR, TG, TEM, and XPS results confirmed the successful covalently modification of HNTs by PMMA. PVC/PMMA-grafted HNTs nanocomposites have been prepared by melt compounding. The PMMA-grafted HNTs could improve the toughness, strength, modulus of PVC nanocomposites simultaneously. TEM and SEM observations revealed that the PMMA-grafted HNTs uniformly dispersed in the PVC matrix. The fracture surfaces of the nanocomposites exhibited plastic deformation feature indicating ductile fracture behaviors. The improvement of toughness of PVC by PMMAgrafted HNTs was attributed to the improved interfacial bonding by grafting and the toughening mechanism was explained according to the cavitation mechanism.

\section{Acknowledgements}

The authors would like to acknowledge the XinJiang Tianye Group Co., Ltd.

\section{References}

[1] Wan C. Y., Qiao X. Y., Zhang Y., Zhang Y. X.: Effect of different clay treatment on morphology and mechanical properties of PVC-clay nanocomposites. Polymer Testing, 22, 453-461 (2003). DOI: 10.1016/s0142-9418(02)00126-5

[2] Xie X-L., Liu Q-X., Li R. K-Y., Zhou X-P., Zhang QX., Yu Z-Z., Mai Y-W.: Rheological and mechanical properties of $\mathrm{PVC} / \mathrm{CaCO}_{3}$ nanocomposites prepared by in situ polymerization. Polymer, 45, 6665-6673 (2004).

DOI: $10.1016 /$ j.polymer.2004.07.045

[3] Wang G. J., Wang L. J., Mei Z., Chang Z. M.: Reinforcement and toughening of poly(vinyl chloride) with poly(caprolactone) grafted carbon nanotubes. Composites Part A: Applied Science and Manufacturing, 40, 1476-1481 (2009).

DOI: 10.1016/j.compositesa.2009.05.011

[4] Xie X-L., Li R. K-Y., Liu Q-X., Mai Y-W.: Structureproperty relationships of in-situ PMMA modified nano-sized antimony trioxide filled poly(vinyl chloride) nanocomposites. Polymer, 45, 2793-2802 (2004). DOI: $10.1016 /$ j.polymer.2004.02.028

[5] Zhu A. P., Cai A. Y., Zhang J., Jia H. W., Wang J. Q.: PMMA-grafted-silica/PVC nanocomposites: Mechanical performance and barrier properties. Journal of Applied Polymer Science, 108, 2189-2196 (2008). DOI: $10.1002 /$ app. 27863

[6] Xie X. L., Li B. G., Pan Z. R., Li R. K. Y., Tjong S. C.: Effect of talc/MMA in situ polymerization on mechanical properties of PVC-matrix composites. Journal of Applied Polymer Science, 80, 2105-2112 (2001). DOI: 10.1002/app.1310

[7] Du M. L., Guo B. C., Jia D. M.: Thermal stability and flame retardant effects of halloysite nanotubes on poly(propylene). European Polymer Journal, 42, 13621369 (2006).

DOI: $\underline{10.1016 / j . e u r p o l y m j .2005 .12 .006}$ 
[8] Liu M. X., Guo B. C., Du M. L., Chen F., Jia D. M.: Halloysite nanotubes as a novel $\beta$-nucleating agent for isotactic polypropylene. Polymer, 50, 3022-3030 (2009).

DOI: 10.1016/j.polymer.2009.04.052

[9] Deng S. Q., Zhang J. N., Ye L., Wu J. S.: Toughening epoxies with halloysite nanotubes. Polymer, 49, 51195127 (2008).

DOI: $10.1016 /$ j.polymer.2008.09.027

[10] Ye Y. P., Chen H. B., Wu J. S., Ye L.: High impact strength epoxy nanocomposites with natural nanotubes. Polymer, 48, 6426-6433 (2007). DOI: $10.1016 /$ j.polymer.2007.08.035

[11] Liu M. X., Guo B. C., Du M. L., Cai X. J., Jia D. M.: Properties of halloysite nanotube-epoxy resin hybrids and the interfacial reactions in the systems. Nanotechnology, 18, 455703/1-455703/9 (2007). DOI: $10.1088 / 0957-4484 / 18 / 45 / 455703$

[12] Jia Z. X., Luo Y. F., Guo B. C., Yang B. T., Du M. L.: Reinforcing and flame-retardant effects of halloysite nanotubes on LLDPE. Polymer-Plastics Technology and Engineering, 48, 607-613 (2009).

DOI: $10.1080 / 03602550902824440$

[13] Guo B. C., Zou Q. L., Lei Y. D., Jia D. M.: Structure and performance of polyamide 6/halloysite nanotubes nanocomposites. Polymer Journal, 41, 835-842 (2009). DOI: $10.1295 /$ polymj.PJ2009110

[14] Guo B. C., Zou Q. L., Lei Y. D., Du M. L., Liu M. X., Jia D. M.: Crystallization behavior of polyamide 6/halloysite nanotubes nanocomposites. Thermochimica Acta, 484, 48-56 (2009).

DOI: $10.1016 /$ j.tca.2008.12.003

[15] Rooj S., Das A., Thakur V., Mahaling R. N., Bhowmick A. K., Heinrich G.: Preparation and properties of natural nanocomposites based on natural rubber and naturally occurring halloysite nanotubes. Materials and Design, 31, 2151-2156 (2010). DOI: $10.1016 / \mathrm{j}$. matdes.2009.11.009

[16] Du M. L., Guo B. C., Lei Y. D., Liu M. X., Jia D. M.: Carboxylated butadiene-styrene rubber/halloysite nanotube nanocomposites: Interfacial interaction and performance. Polymer, 49, 4871-4876 (2008). DOI: $10.1016 /$ j.polymer.2008.08.042

[17] Pasbakhsh P., Ismail H., Fauzi M. N. A., Abu Bakar A.: Influence of maleic anhydride grafted ethylene propylene diene monomer (MAH-g-EPDM) on the properties of EPDM nanocomposites reinforced by halloysite nanotubes. Polymer Testing, 28, 548-559 (2009). DOI: $10.1016 /$ j.polymertesting.2009.04.004

[18] Mondragón M., Roblero-Linares Y. S., SánchezEspindola M. E., Zendejas-Leal B. E.: Rigid poly(vinyl chloride)/halloysite nanocomposites. in 'Nanotech conference and expo 2009, Technical proceedings - Nanotechnology 2009: Life Sciences, medicine, diagnostics, bio materials and composites. Boca Raton, USA, 482-484 (2009).
[19] Zhang Y. W., Jiang J. Q., Liang Q. H., Zhang B.: Modification of halloysite nanotubes with poly(styrenebutyl acrylate-acrylic acid) via in situ soap-free graft polymerization. Journal of Applied Polymer Science, 117, 3054-3059 (2010).

DOI: $10.1002 /$ app.32087

[20] Wan C. Y., Li M., Bai X., Zhang Y.: Synthesis and characterization of photoluminescent Eu(III) coordination halloysite nanotube-based nanohybrids. Journal of Physical Chemistry C, 113, 16238-16246 (2009). DOI: $10.1021 /$ jp9051648

[21] Moulder J. F., Stickle W. F., Sobol P. E., Bomben K. D.: Handbook of X ray photoelectron spectroscopy. Perkin-Elmer Corporation, Minnesota (1992).

[22] Liu M. X., Guo B. C., Du M. L., Lei Y. D., Jia D. M.: Natural inorganic nanotubes reinforced epoxy resin nanocomposites. Journal of Polymer Research, 15, 205-212 (2008). DOI: $10.1007 / \mathrm{s} 10965-007-9160-4$

[23] Meng X. Y., Wang Z., Tang T.: Controlling dispersed state and exfoliation process of clay in polymer matrix. Materials Science and Technology, 22, 780-786 (2006). DOI: $10.1179 / 174328406 \times 101238$

[24] Manchado M. A. L., Valentini L., Biagiotti J., Kenny J. M.: Thermal and mechanical properties of singlewalled carbon nanotubes-polypropylene composites prepared by melt processing. Carbon, 43, 1499-1505 (2005).

DOI: $10.1016 /$ j.carbon.2005.01.031

[25] Yang C. J., Gong C. Q., Peng T. Y., Deng K. J., Zan L.: High photocatalytic degradation activity of the polyvinyl chloride (PVC)-vitamin $\mathrm{C}(\mathrm{VC})-\mathrm{TiO}_{2}$ nano-composite film. Journal of Hazardous Materials, 178, 152-156 (2010).

DOI: $10.1016 /$ j.jhazmat.2010.01.056

[26] Gojny F. H., Wichmann M. H. G., Fiedler B., Schulte $\mathrm{K}$.: Influence of different carbon nanotubes on the mechanical properties of epoxy matrix composites - A comparative study. Composites Science and Technology, 65, 2300-2313 (2005). DOI: 10.1016/j.compscitech.2005.04.021

[27] Awad W. H., Beyer G., Benderly D., Ijdo W. L., Songtipya P., Jimenez-Gasco M. D., Manias E., Wilkie C. A.: Material properties of nanoclay PVC composites. Polymer, 50, 1857-1867 (2009). DOI: $10.1016 /$ j.polymer.2009.02.007

[28] Sheng S. S., Ling Z., Zhong L. C., Ming C. H., Ling Z. Q.: Effect of properties of $\mathrm{CaCO}_{3}$ nanoparticles on interface and properties of poly (vinyl chloride) composites (in Chinese). Journal of Chemical Industry and Engineering, 56, 2233-2239 (2005).

[29] Peng S. W., Wang X. Y., Dong L. S.: Special interaction between poly (propylene carbonate) and corn starch. Polymer Composites, 26, 37-41 (2005). DOI: $10.1002 /$ pc. 20070 
[30] Jancar J.: Influence of filler particle shape on elastic moduli of $\mathrm{PP} / \mathrm{CaCO}_{3}$ and $\mathrm{PP} / \mathrm{Mg}(\mathrm{OH})_{2}$ composites, Part 1 'Zero' interfacial adhesion. Journal of Materials Science, 24, 3947-3955 (1989). DOI: $10.1007 / \mathrm{BF} 01168958$

[31] Wan C. Y., Tian G. H., Cui N., Zhang Y. X., Zhang Y.: Processing thermal stability and degradation kinetics of poly(vinyl chloride)/montmorillonite composites. Journal of Applied Polymer Science, 92, 1521-1526 (2004).

DOI: $10.1002 /$ app.20086
[32] Gong F. L., Feng M., Zhao C. G., Zhang S. M., Yang M. S.: Thermal properties of poly(vinyl chloride)/ montmorillonite nanocomposites. Polymer Degradation and Stability, 84, 289-294 (2004).

DOI: $10.1016 /$ j.polymdegradstab.2003.11.003

[33] Zuiderduin W. C. J., Westzaan C., Huetink J., Gaymans R. J.: Toughening of polypropylene with calcium carbonate particles. Polymer, 44, 261-275 (2003).

DOI: 10.1016/S0032-3861(02)00769-3

[34] Olabisi O., Robeson L. M., Shaw M. T.: Polymerpolymer miscibility. Academic Press, New York (1979) 